\title{
Analytic understanding and control of dynamical friction
}

\author{
Emanuele Panizon, ${ }^{1}$ Giuseppe E. Santoro, ${ }^{1,2}$ Erio Tosatti, ${ }^{1,2,3}$ Gabriele Riva, ${ }^{4}$ and Nicola Manini ${ }^{4}$ \\ ${ }^{1}$ International School for Advanced Studies, Via Bonomea 265, 34136 Trieste, Italy \\ ${ }^{2}$ International Center for Theoretical Physics (ICTP), Strada Costiera 11, I-34151 Trieste, Italy \\ ${ }^{3}$ CNR-IOM Democritos National Simulation Center, Via Bonomea 265, 34136 Trieste, Italy \\ ${ }^{4}$ Dipartimento di Fisica, Università degli Studi di Milano, Via Celoria 16, 20133 Milano, Italy
}

(Received 31 January 2018; published 15 March 2018)

\begin{abstract}
Recent model simulations discovered unexpected nonmonotonic features in the wear-free dry phononic friction as a function of the sliding speed. Here we demonstrate that a rather straightforward application of linear-response theory, appropriate in a regime of weak slider-substrate interaction, predicts frictional one-phonon singularities which imply a nontrivial dependence of the dynamical friction force on the slider speed and/or coupling to the substrate. The explicit formula which we derive reproduces very accurately the classical atomistic simulations when available. By modifying the slider-substrate interaction the analytical understanding obtained provides a practical means to tailor and control the speed dependence of friction with substantial freedom.
\end{abstract}

DOI: 10.1103/PhysRevB.97.104104

\section{INTRODUCTION}

Friction is ubiquitous for contacting objects in relative motion. Macroscopic mechanical energy gets converted more or less rapidly, besides wear and other structural transformations, into thermal energy, namely the random excitations of the vibrational degrees of freedom (phonons) of solids, as well as electronic ones, when available. Dissipation occurs from the largest scale of sliding geological faults down to the nanometric and atomic scale of nanoelectromechanical devices and atomic force microscopy (AFM) experiments. Following the great variety of experimental and technological approaches, the fundamentals of friction have been hystorically well rationalized [1-9]. Nonetheless, the corresponding theoretical efforts addressing the underlying physics are not yet complete and satisfactory, and many aspects still require clarification. In particular, basic standard models introduced in the early 20th century, namely the Prandtl-Tomlinson model [10,11] and the Frenkel-Kontorova model [12-15], are still largely used to date as workhorses representing phononic, wear-free static, and sliding friction at an atomic level. In addition, current theoretical research relies routinely on system-specific models for molecular-dynamics (MD) simulations based on realistic interatomic force fields. Both the early models and the modern MD simulations usually rely on adding dissipation to the energy-conserving atomic dynamics by means of artificially added thermostats of some kind, most often a Langevin thermostat [16]. Depending on the property investigated, this kind of approach may be acceptable or problematic. In general, since frictional dissipation transforms the mechanical work into heat, one should worry about spurious effects brought in by the thermostat, whose job is precisely to remove that extra heat. After all, if the rate of frictional dissipation is what one wishes to describe, then the results may not be unaffected by the arbitrary downstream energy dissipation put in "by hand" by the thermostat. The present paper aims to provide a step forward in this understanding of friction, by means of an analytic insight based on linear-response theory (LRT). Once a reliable analytic understanding of friction is obtained, even though in a simple model, it will inevitably suggest ways to control it and tune it with a certain freedom.

As mentioned above, the classical frictional force and power dissipation by a slider moving on a substrate is-in a regime where the coupling is weak and wear and nonlinear stick-slip phenomena are absent-essentially due to the excitation of phonons. If the slider can be considered rigid, the relevant phonon spectrum $\omega(Q)$ is that of the substrate. The slidersubstrate interaction potential, or better its Fourier transform $V_{\text {ext }}(Q)$, will in turn determine the strength of the coupling generating such phonons. In the weak interaction regime, multiphonon processes are negligible and the excitation of single phonons dominates the inelastic energy loss, yielding in principle a great predictability to the resulting friction. The frictional force $F$ will in this case be controlled by detailed resonances which depend on the slider velocity $v_{\mathrm{SL}}$, on the phonon spectrum $\omega(Q)$, and on the coupling potential $V_{\text {ext }}(Q)$, giving rise, as we will show, to a nontrivial nonmonotonic behavior quite different from the macroscopic friction laws.

In order to exemplify this physical process, we focus on the simplest model [17] — a point slider moving at velocity $v_{\mathrm{SL}}$ interacting weakly with a harmonic chain underneath, see sketch in Fig. 1(a), through van-der-Waals forces. Numerical simulations of this model revealed a nontrivial dependence of friction on the sliding speed [17]. In the following, we will develop the analytic formula for the phononic friction in the linear-response approximation, thus providing a much more general handle on the prediction of friction at the nanoscale. The theory which we will set up within LRT, appropriate for the assumed weak slider-solid interaction, is relatively simple but remarkably rich of consequences. The final result for the average friction force $F$ felt by the slider can be cast in the 

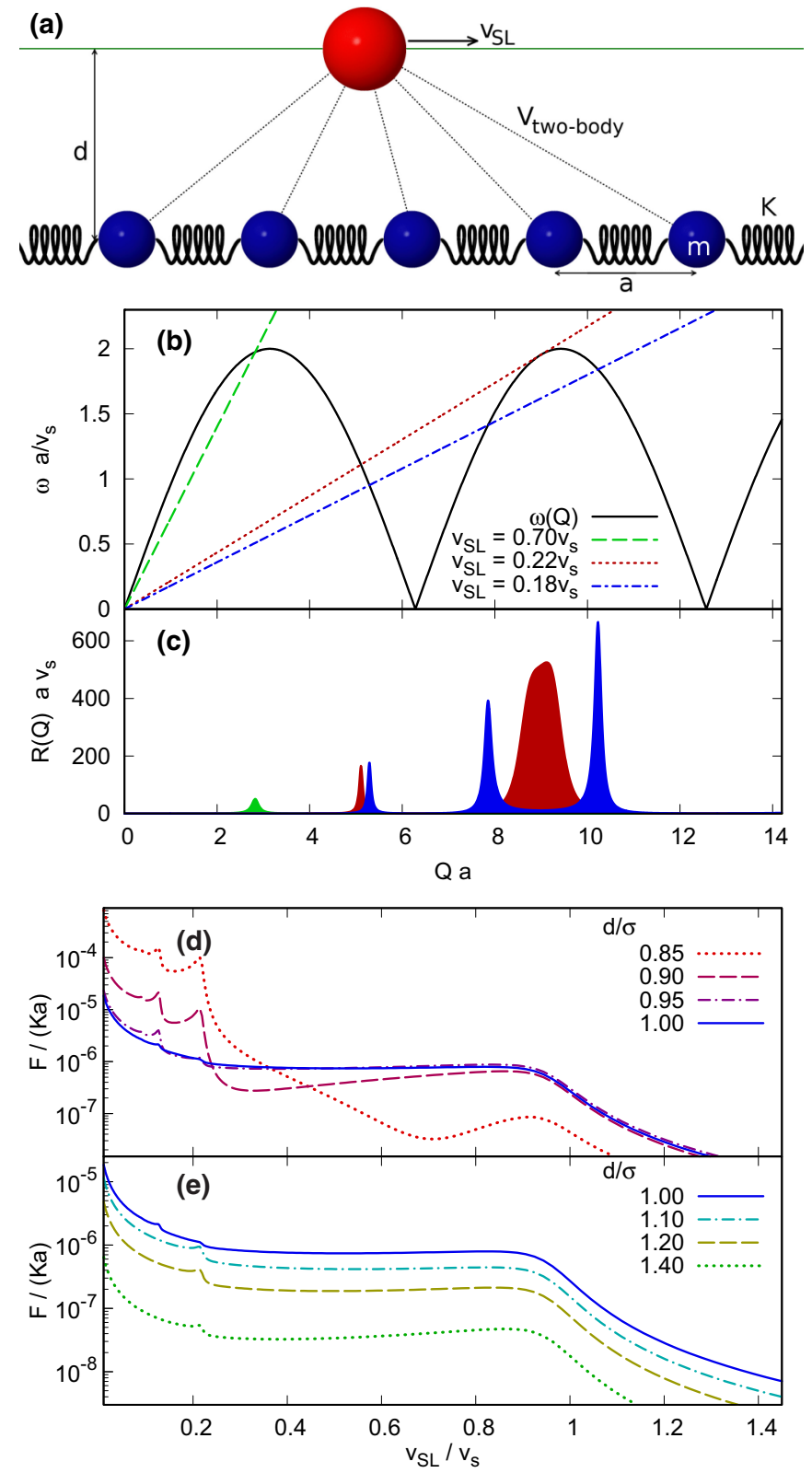

FIG. 1. (a) A sketch of the one-dimensional model [17] considered in this paper. (b) Graphical solutions of the energy-conservation condition $v_{\mathrm{SL}} Q=\omega(Q)$ which determines the inelastically excited phonons for three different slider speeds $v_{\mathrm{SL}}$ expressed in units of the sound velocity $v_{\mathrm{s}}$. (c) The resonant magnitude evaluated as the term $R(Q)=\gamma /(2 \pi) Q^{2} /\left\{\left[Q v_{\mathrm{SL}}-\omega(Q)\right]^{2}+(\gamma / 2)^{2}\right\}$ of Eq. (1). (d),(e) Friction as a function of $v_{\mathrm{SL}}$ at several distances $d$ between the slider and the chain. $V_{\text {ext }}$ is defined by a Lennard-Jones potential with $\sigma=a / 2$ and $V_{0}=5 \times 10^{-4} \mathrm{Ka}^{2}$. The harmonic chain particle motion has a damping coefficient $\gamma=0.1(m / K)^{1 / 2}$. Parameters $a$, $m$, and $K$ are the spacing, mass, and spring constant of the harmonic chain, as indicated in panel (a).

illuminating form:

$$
\begin{aligned}
F\left(v_{\mathrm{SL}}\right)= & \frac{1}{2 m a v_{\mathrm{SL}}} \int_{0}^{+\infty} d Q Q^{2}\left|V_{\mathrm{ext}}(Q)\right|^{2} \\
& \times \frac{1}{\pi} \frac{\gamma / 2}{\left[Q v_{\mathrm{SL}}-\omega(Q)\right]^{2}+(\gamma / 2)^{2}},
\end{aligned}
$$

where $v_{\mathrm{SL}}$ is the slider velocity, $\omega(Q)$ the dispersion of the sound modes of the harmonic chain, and $V_{\text {ext }}(Q)$ the Fourier transform of the slider-solid interaction potential. Here $\gamma$ is a small frictional damping constant which affects the motion of each chain particle, representing all other degrees of freedom coupling to the chain phonons: It results in a finite lifetime of the phonons themselves, giving rise to a Lorentzian smearing of the resonances that occur when the slider velocity $v_{\mathrm{SL}}$ matches the phase velocity $\omega(Q) / Q$ of the phonons. Note that the integral over $Q$ implies an extended brillouin zone (BZ) scheme for the phonons, thus not limited within $[-\pi / a, \pi / a]$. While at very large speeds there are no solutions to the resonance condition $Q v_{\mathrm{SL}}=\omega(Q)$, as $v_{\mathrm{SL}}$ falls below the harmonicchain speed of sound $v_{\mathrm{s}}$ one or more solutions appear, as illustrated in Figs. 1(b) and 1(c). Sharp resonance conditions occur at critical velocities $v_{\mathrm{SL}}$ that match the group velocity $v(Q)=$ $d \omega / d Q$ at special wave vectors $Q_{i}$, an event which gives rise to a van Hove singularity in the integrand, and a consequently sharp increase of the friction. Remarkably, the overall shape of $F\left(v_{\mathrm{SL}}\right)$ depends only weakly on the small damping coefficient $\gamma$, which simply provides a smearing of the singularities. The crucial weighting factor in the integral is the slider-chain interaction potential Fourier transform $\left|V_{\text {ext }}(Q)\right|^{2}$, which in practice could be manipulated by, e.g., modifying the slider shape and/or its distance $d$ from the substrate. Figures 1(d) and 1(e) shows how nonmonotonic and complex the dynamical friction force $F$ can result, as a function of the slider velocity $v_{\mathrm{sL}}$ and of the distance $d$ from the substrate.

This paper is organized as follows. In Sec. II we introduce the model, previously simulated numerically in Ref. [17], and recall briefly a few tools of LRT. We then apply them to derive the explicit decomposition of the friction force into products of equilibrium dynamical properties of the unperturbed chain and mechanical properties of the slider-chain interaction. This decomposition is then used in Sec. III to evaluate the dynamical friction force as a function of the sliding velocity for the model at hand. The striking parameter-free agreement of the analytical results with previous MD simulations is presented and qualified. The effects of the thermostat dissipation in the chain is also discussed. In Sec. IV we then take advantage of the simplicity and flexibility of the analytical result (1) to investigate how changes in the slider-chain interaction potential affect the dissipation profile, thus providing ways to tune friction, and in particular its dependence on speed. Conclusions are drawn in Sec. V.

\section{MODEL AND LINEAR-RESPONSE THEORY}

The model we consider in this paper was introduced and described in Ref. [17]. As sketched in Fig. 1(a), it consists of a slider, implemented in its simplest form as a pointlike particle characterized by mass $M$, position $x_{\mathrm{SL}}$, and velocity $v_{\mathrm{sL}}$, interacting weakly via a two-body potential with each atom in a harmonic chain characterized by particles of mass $m$, nearest-neighbor couplings with spring constant $K$, and equilibrium spacing $a$. The slider and the chain atoms move in one dimension (1D) along parallel lines at a fixed distance $d$. The slider-chain interaction energy is modeled by a sum of two-body terms, $\sum_{j} V_{\text {two-body }}\left(\sqrt{\left|x_{j}-x_{\mathrm{SL}}\right|^{2}+d^{2}}\right)$. $V_{\text {two-body }}$ is 
taken, e.g., as a $(12,6)$ Lennard-Jones (LJ) function with the minimum of depth $V_{0}$ at a separation $\sigma$.

We proceed next by assuming that the interaction between slider and chain is weak. Under this condition, each "collision" occurring as the slider comes close to particles in the chain leads to a negligible change of the slider's momentum and kinetic energy. As in the Born approximation of scattering theory, the slider motion is thus conveniently approximated by an unperturbed free motion $x_{\mathrm{SL}}=v_{\mathrm{SL}} t$. This is only meaningful as long as the typical interaction strength $V_{0}$ is much smaller than the kinetic energy of the slider $M v_{\mathrm{sL}}^{2} / 2$, a condition that can be reformulated as $v_{\mathrm{SL}} \gg\left(V_{0} / M\right)^{1 / 2}$. In this approximation, the $j$ th particle of the harmonic chain is influenced by a weak time-dependent external potential $V_{\text {ext }}\left(x_{j}, t\right)=$ $V_{\text {two-body }}\left(\sqrt{\left|x_{j}-v_{\text {SL }} t\right|^{2}+d^{2}}\right)$. We can then express the total Hamiltonian of the weakly perturbed harmonic chain as:

$$
H_{\text {chain }}(t)=H_{\text {harm }}+\int_{-\infty}^{+\infty} d x V_{\text {ext }}(x, t) n(x),
$$

where $H_{\text {harm }}$ is the Hamiltonian of the unperturbed harmonic chain. Here $V_{\text {ext }}(x, t)$ is the (small) amplitude of the perturbation, and the density $n(x)=\sum_{j} \delta\left(x-x_{j}\right)$ is the corresponding "operator" to which it couples. This formulation lends itself ideally to use LRT $[18,19]$. Technically, we will adopt a quantum approach at the start, which we find more convenient: The corresponding classical LRT approach would amount to the substitution of the quantum von-Neumann equation for the density matrix with the corresponding classical Liouville equation [18]. In the following we outline this calculation without omitting any useful detail, but in lighter form-more mathematical derivations are provided in the Appendices.

As in Ref. [17], we calculate the dynamical friction force $F$ generated by sliding, which can be immediately related to the dissipated power $W=F v_{\mathrm{SL}}$. Let us focus on the internal energy $E(t)$ of the perturbed chain. LRT tells us that [19]:

$$
\begin{aligned}
\frac{d}{d t} E(t) \simeq & -\int_{-\infty}^{+\infty} d x \int_{-\infty}^{+\infty} d x^{\prime} \int_{-\infty}^{+\infty} d t^{\prime} \\
& \times V_{\mathrm{ext}}(x, t) \frac{\partial \chi_{n n}^{R}\left(x, x^{\prime} ; t-t^{\prime}\right)}{\partial t} V_{\mathrm{ext}}\left(x^{\prime}, t^{\prime}\right),
\end{aligned}
$$

where $\chi_{n n}^{R}\left(x, x^{\prime} ; t-t^{\prime}\right)=-\frac{i}{\hbar} \theta\left(t-t^{\prime}\right)\left\langle\left[\hat{n}(x, t), \hat{n}\left(x^{\prime}, t^{\prime}\right)\right]\right\rangle$ is the retarded density-density response function, the average $\langle\cdots\rangle$ taken on the equilibrium Gibbs ensemble, and $V_{\mathrm{ext}}(x, t)$ is a weak but arbitrary external potential, assumed to depend only on $\left(x-v_{\mathrm{SL}} t\right)$. The instantaneous power dissipated by the slider equals the rate of increase of the chain internal energy $W=d E / d t$. To evaluate the mean friction force $F$ opposing the slider motion, this power $W$ must be averaged over a period $\tau=a / v_{\mathrm{SL}}$, which is the natural "washboard time" for the slider moving across a substrate with a corrugation of period $a: F=\bar{W} / v_{\mathrm{sL}}$. To address this periodic problem it is advantageous to work in the $(Q, \omega)$ Fourier domain. In this way we can enforce the crystalline translational invariance, leading to (see Appendix A for details) a simpler expression for the average friction force in terms of the imaginary part of the density-density susceptibility, or equivalently, via the fluctuation-dissipation theorem [19], of the corresponding dynamical structure factor:

$$
\begin{aligned}
F\left(v_{\mathrm{SL}}\right) & =-\frac{2}{v_{\mathrm{SL}}} \int_{0}^{+\infty} \frac{d Q}{2 \pi} \widetilde{\omega}_{Q} \operatorname{Im} \chi_{n n}^{R}\left(Q, Q ; \widetilde{\omega}_{Q}\right)\left|V_{\mathrm{ext}}(Q)\right|^{2} \\
& =\int_{0}^{+\infty} \frac{d Q}{2 \pi} Q\left(1-\mathrm{e}^{-\beta \hbar \widetilde{\omega}_{Q}}\right) S_{n n}\left(Q, Q ; \widetilde{\omega}_{Q}\right)\left|V_{\mathrm{ext}}(Q)\right|^{2} .
\end{aligned}
$$

As required by momentum conservation in all such energy-loss problems, in Eq. (4) only frequencies $\widetilde{\omega}_{Q}=v_{\text {sL }} Q$ contribute. Equation (4) realizes a decomposition of the friction force into products of the Fourier components of the structure factor of the unperturbed chain at thermodynamic equilibrium, and of squared Fourier components of the slider-chain interaction potential. On one hand, this kind of decomposition is quite standard in all applications of the LRT, as in the ordinary Born approximation of scattering theory. Application of this method to a proper sliding-friction problem was rather rarely attempted before (see however Refs. [20,21]). The present application demonstrating an exceptionally high accuracy of the results represents a major milestone of the present paper. The reader must be warned that the strict applicability of LRT is limited to smooth-sliding regimes where the system remains clear of highly nonlinear effects such as stick-slip dynamics or wear, which can and do occur in the physics of friction $[2,8,9,22-$ 26]. In the present weak-perturbation approach however, the Prandtl-Tomlinson smooth-sliding condition [27] is always automatically satisfied. For smooth sliding therefore, one can take advantage of the analytical predictive power of the LRT decomposition, as we shall illustrate in the following sections.

\section{EVALUATION OF THE DYNAMICAL FRICTION FORCE}

We now apply the general LRT prescription in Eq. (4)which with minor adjustments would describe more realistic slider-substrate situations in higher dimensions-to our toy problem of a 1D harmonic chain substrate perturbed by a point slider a distance $d$ away. The exact expression for the harmonicchain structure factor $S_{n n}(Q, Q, \omega)$-see Appendix B for a derivation -is reported in Eq. (B6) but is practically impossible to use. To proceed, we make use of a relatively standard one-phonon approximation [28], which is quite reasonable in higher dimensions, ignoring here all pathologies typical of $1 \mathrm{D}$, due in turn to singularities generated by the $1 / \omega(Q)$ factor appearing in the $Q$ integrals. Taken literally—see Appendix B for a discussion of the relevant steps and subtleties-the onephonon approximation leads to the following expression for the dynamical structure factor:

$$
S_{n n}^{1-\mathrm{ph}}(Q, Q ; \omega>0)=\frac{\pi Q^{2}}{m a \omega(Q)} \frac{1}{1-\mathrm{e}^{-\beta \hbar \omega(Q)}} \delta(\omega-\omega(Q)),
$$

where $\omega(Q)$ is the phonon dispersion in the extended BZ scheme. Substituting this approximate structure factor in the expression for the friction force, Eq. (4), we obtain:

$$
\begin{aligned}
F\left(v_{\mathrm{SL}}\right)= & \int_{0}^{+\infty} \frac{d Q}{2 \pi} Q\left(1-\mathrm{e}^{-\beta \hbar v_{\mathrm{SL}} Q}\right) \frac{\pi Q^{2}}{m a \omega(Q)} \frac{1}{1-\mathrm{e}^{-\beta \hbar \omega(Q)}} \\
& \times \delta\left(v_{\mathrm{SL}} Q-\omega(Q)\right)\left|V_{\mathrm{ext}}(Q)\right|^{2} .
\end{aligned}
$$




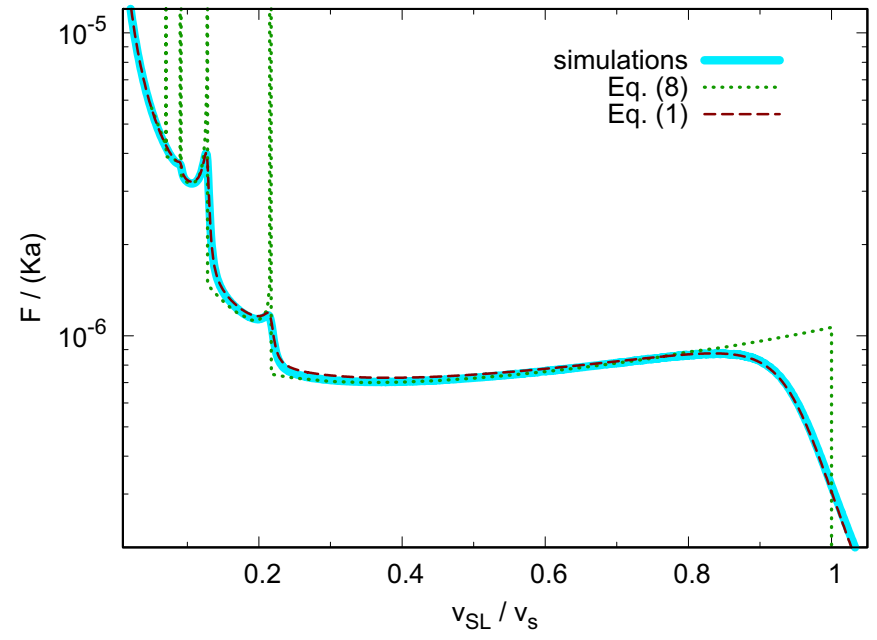

FIG. 2. Comparison of the slider-speed dependence of the friction force $F$ obtained from the two analytical expressions (8) (dotted, no dissipation included) and (1) (dashed, dissipation included), with that obtained for the same conditions by numerical simulations carried out for a chain of 500 atoms (solid) [17]. All calculations have spacing $a$, nearest-neighbor spring constant $K$, damping rate $\gamma=0.1(\mathrm{~m} / K)^{1 / 2}$, LJ slider chain interaction with $\sigma=0.5 a$, and distance $d=0.475 a$. Peaks for $v_{\mathrm{SL}} \leqslant 0.22 v_{\mathrm{s}}$ correspond to speeds listed in Table I and evolve from true divergences without dissipation to sharp peaks with dissipation. The agreement between the analytical and the simulation results is genuinely striking.

The integral over $Q$ is now easy to perform using the wellknown property of the Dirac delta:

$$
\int_{-\infty}^{\infty} d Q g(Q) \delta(f(Q))=\sum_{i} \frac{g\left(Q_{i}\right)}{\left|f^{\prime}\left(Q_{i}\right)\right|},
$$

where $Q_{i}$ are the solutions of $f(Q)=0$. In the present case, $f(Q)=v_{\mathrm{SL}} Q-\omega(Q)$ and $f^{\prime}(Q)=v_{\mathrm{SL}}-v(Q)$, where $v(Q)=d \omega / d Q$ is the group velocity of the phonon dispersion. Substituting and simplifying, we conclude that:

$$
F\left(v_{\mathrm{SL}}\right)=\frac{1}{2 m a v_{\mathrm{SL}}} \sum_{i} \frac{Q_{i}^{2}}{\left|v_{\mathrm{SL}}-v\left(Q_{i}\right)\right|}\left|V_{\mathrm{ext}}\left(Q_{i}\right)\right|^{2} .
$$

Here the values $Q_{i}$ are the solutions of the momentumconservation equation

$$
v_{\mathrm{SL}} Q=\omega(Q) .
$$

Note that both $\hbar$ and the inverse temperature $\beta$, which still enter the expression for $S_{n n}^{1-p h}(Q, Q ; \omega>0)$, have remarkably disappeared from the final expression for $F\left(v_{\mathrm{SL}}\right)$. The expression (8) for $F\left(v_{\mathrm{SL}}\right)$ compares in surprisingly accurate detail against earlier classical numerical simulations for the 1D toy problem [17], see Fig. 2. Indeed all the important frictional features - the overall nonresonant friction, and the positions and relative strengths of the resonant peaks — are reproduced. The overall quantitative agreement is impressive, except for the divergences corresponding to the vanishing denominators in Eq. (8), which in the simulation are replaced by rounded peaks.

The reason for the smoothing of singularities can be attributed to the presence of a (small but finite) viscous force $-\gamma \dot{x}_{j}$ introduced in the classical numerical simulations [17] in order to dispose of the phonon energy generated by the slider before they return to the contact point through the boundary conditions. We expect that in general the unavoidable presence of dissipation and anharmonicity in the substrate will lead to a decay of the density-density correlation function for large $t$ :

$$
S_{n n}^{\text {diss }}\left(x, x^{\prime} ; t\right)=S_{n n}\left(x, x^{\prime} ; t\right) \mathrm{e}^{-\frac{\gamma}{2}|t|} .
$$

This decay will in turn lead to a broadening of the $\delta(\omega-\omega(Q))$ appearing in the one-phonon structure factor in Eq. (5):

$$
\delta(\omega-\omega(Q)) \rightarrow \frac{1}{\pi} \frac{\gamma / 2}{(\omega-\omega(Q))^{2}+(\gamma / 2)^{2}} .
$$

Hence, accounting for dissipation one obtains the friction-force expression (1). This final expression is possibly even more straightforward for a numerical evaluation than Eq. (8). As shown by the dashed line in Fig. 2, the resulting friction reproduces quantitatively the simulated results at all speeds with no fitting parameter, taking for the damping rate the same value $\gamma=0.1(\mathrm{~m} / \mathrm{K})^{1 / 2}$ used in the simulations [17].

We now discuss the physical insights present in the expressions Eq. (1) and Eq. (8). Let us focus, for clarity of presentation, on the latter, which is slightly more transparent in notation, while qualitatively similar to the former. We observe first that at any given speed $v_{\mathrm{SL}}$ all contributions to friction come from a small set of phonon modes which are the solutions of the relation (9), $v_{\mathrm{SL}} Q=\omega(Q)$, equating the slider speed to the phase velocity of the phonons [17]. For nearest-neighbor springs the chain dispersion relation is $\omega(Q)=2 v_{\mathrm{s}} a^{-1}|\sin (Q a / 2)|$. The condition $v_{\mathrm{SL}} Q=\omega(Q)$ is thus conveniently rewritten in dimensionless form

$$
\bar{v}_{\mathrm{SL}} \bar{Q}=2|\sin (\bar{Q} / 2)|,
$$

where $\bar{Q}=Q a$ and $\bar{v}_{\mathrm{SL}}=v_{\mathrm{SL}} / v_{\mathrm{s}}$ is the ratio between the slider speed and the speed of sound $v_{\mathrm{s}}=a(K / m)^{1 / 2}$, and we introduce the dimensionless dispersion $\bar{\omega}(\bar{Q})=a v_{\mathrm{s}}^{-1} \omega(\bar{Q} / a)=$ $2|\sin (\bar{Q} / 2)|$.

For a given (dimensionless) slider speed $\bar{v}_{\mathrm{SL}}$, each intersection of the straight line $\bar{v}_{\mathrm{SL}} \bar{Q}$ with the phonon dispersion $\bar{\omega}(\bar{Q})$ determines, regardless of details of the sliderchain interaction, a contribution to friction. This is illustrated in Fig. 1(b) for three velocities. For $\bar{v}_{\mathrm{SL}}>1$, the resonant phonon intersections cease, and Eq. (8) predicts frictionless sliding, as reflected by the sharp drop of the dotted line in Fig. 2. As $\bar{v}_{\mathrm{SL}}$ decreases below unity, initially Eq. (9) has a single solution $\bar{Q}_{1}$ in the $[0,2 \pi)$ interval, as for the dashed line in Fig. 1(b). Then, starting from $\bar{v}_{\text {SL }} \lesssim 0.217$, two new solutions appear at $2 \pi<\bar{Q}_{2} \leqslant \bar{Q}_{3}<4 \pi$, as for the dotted and dot-dashed lines in Fig. 1(b). More solutions appear in pairs at larger $\bar{Q}$ as $\bar{v}_{\mathrm{SL}}$ is further reduced-therefore friction grows.

According to Eq. (8), friction is determined by the magnitude of the squared Fourier transform of the external potential at the resonating wave vectors $Q_{i}=\bar{Q}_{i} / a$. These magnitudes are summed with weights given by $Q_{i}^{2} /\left|v_{\mathrm{SL}}-v\left(Q_{i}\right)\right|$. This leads to the emergence of sharp resonance conditions when $v\left(Q_{i}\right) \sim v_{\mathrm{SL}}$, similar to those reported earlier in the FrenkelKontorova model $[14,29,30]$. The resulting divergences in $F$ resemble van Hove singularities [31] in 1D. The regular van Hove singularities, e.g., in the density of states, are associated 
TABLE I. Resonant dimensionless velocities $\bar{v}_{\mathrm{SL} j}$, and corresponding solutions $\bar{Q}_{j}=Q_{j} a$ for the first ten friction peaks for decreasing $\bar{v}_{\mathrm{SL}}=v_{\mathrm{SL}} / v_{\mathrm{s}}$, evaluated from the tangency condition, Eq. (13). The last column reports the values of $\bar{Q}_{j}$ folded back to the first BZ $(-\pi, \pi]$.

\begin{tabular}{llcc}
\hline \hline$j$ & \multicolumn{1}{c}{$\bar{v}_{\mathrm{SL} j}$} & $\bar{Q}_{j}$ & $\bar{Q}_{j}(1 \mathrm{st} \mathrm{BZ})$ \\
\hline 1 & 1 & 0 & 0 \\
2 & 0.217243 & 8.986819 & 2.70364 \\
3 & 0.128375 & 15.45050 & 2.88413 \\
4 & 0.091325 & 21.80824 & 2.95869 \\
5 & 0.070914 & 28.13239 & 2.99965 \\
6 & 0.057972 & 34.44151 & 3.02558 \\
7 & 0.049030 & 40.74261 & 3.04349 \\
8 & 0.042480 & 47.03890 & 3.05661 \\
9 & 0.0374745 & 53.33211 & 3.06663 \\
10 & 0.0335251 & 59.62320 & 3.07453 \\
\hline \hline
\end{tabular}

to regions of the BZ where the group velocity vanishes, i.e., $d \omega(Q) / d Q=0$. In the present case the singularities are generated by the condition $Q v_{\mathrm{SL}}=\omega(Q)$, and lead to divergent friction when the slider velocity, the phase velocity, and the group velocity coincide:

$$
v_{\mathrm{SL}}=\frac{\omega(Q)}{Q}=\frac{d \omega(Q)}{d Q} \equiv v(Q) .
$$

Singularities of the same origin are long known in energy-loss problems, such as for example in the crossing of solids by fast electrons [32], or by neutrons [28], or even in inelastic helium scattering at solid surfaces [33].

Here, the resonances have a physically intuitive explanation. The slider moving at speed $v_{\mathrm{SL}}$ can only excite those phonons with matching phase velocity $\omega(Q) / Q$ - their "wave crest" velocity matching that of the slider. However, only when the exciting particle and the excited phonon wave packet, moving with the group velocity $v(Q)$, "fly" together for a long time the energy transfer between them is really strong. Thus the effectiveness of the excited phonons to download and carry energy away is strong-resonant-when all velocities coincide, and weaker when they do not match. Crudely speaking, only at resonance the slider "surfs" the phonon wave crest appropriate for its speed.

Geometrically the divergences predicted occur when the $v_{\mathrm{SL}} Q$ straight line is tangent to the dispersion curve $\omega(Q)$ [such as the dotted line of Fig. 1(b)]: Each divergence corresponds to a solution of Eq. (13), which marks precisely the appearance (disappearance) of a new pair of solutions of $v_{\mathrm{SL}} Q=\omega(Q)$ as $v_{\mathrm{SL}}$ is decreased (increased). Table I reports the numerical values of the ten largest resonant speeds at which such divergences appear.

We stress that these resonant speeds are uniquely functions of the chain dispersion relation, therefore of its structure factor, independent of the "form factor," namely the slider-chain interaction $V_{\text {ext }}$. As for the slider-chain interaction, the theory poses no significant restriction on the shape of the weak potential $V_{\text {ext }}$ beyond that of a dependence on time and space of the form $\left(x-v_{\mathrm{SL}} t\right)$, i.e., $V_{\mathrm{ext}}$ must have a fixed profile translating rigidly at a speed $v_{\mathrm{SL}}$, and it must be possible to evaluate its Fourier transform.

\section{VARYING THE SLIDER-CHAIN INTERACTION}

As shown above, Eq. (1) compares extremely well with MD simulations carried out with a LJ slider-chain potential. There is no reason to believe it would not provide equally reliable friction evaluations for other physically meaningful external potentials. In the following we study the effect on friction of changes in $V_{\text {ext }}$.

We first stick to the basic LJ potential form and investigate the effect of varying the distance $d$ of the slider from the surface. Some interesting novelties that emerge are shown in Figs. 1(d) and 1(e). First of all, as expected, regardless of $d$ the resonant friction peaks occur at the same speeds, listed in Table I, determined purely by the chain dispersion relation. By contrast, the absolute and relative dissipation at the resonances, and in between, do change when $V_{\text {ext }}$ is varied due to changing $d$. We observe in particular a gradual weakening of the low-speed peaks as $d$ is increased. The peaks emerge from solutions of Eq. (13) at large wave vectors $Q$ that correspond to potential variations at very short length scale. As the distance is increased the interaction between slider and substrate smoothens out, large- $Q$ Fourier components decrease dramatically, and therefore only small wave vectors contribute to the dissipation. As a result, at low speed $\bar{v}_{\mathrm{SL}}<0.22$ friction grows monotonically as the perturbation strengthens at shorter distances. By contrast, at larger speed friction shows a nonmonotonic behavior as a function of $d$.

Varying $d$ is somewhat equivalent to varying the load applied, e.g., in an AFM experiment. Since sliding assumes in our model a fixed distance between slider and the substrate line, the vertical (i.e., perpendicular to sliding) force component oscillates in time. For each distance $d$, we can introduce an effective load $L$, as the vertical force experienced by the slider averaged over one period $a / v_{\mathrm{SL}}$, namely

$$
\begin{aligned}
L & =-\frac{v_{\mathrm{SL}}}{a} \int_{0}^{a / v_{\mathrm{SL}}} d t \sum_{j} \frac{d V_{\mathrm{ext}}(j a, t)}{d d} \\
& =-\frac{1}{a} \int_{-\infty}^{\infty} d x \frac{d V_{\mathrm{ext}}(x, 0)}{d d} .
\end{aligned}
$$

$L$ is positive in the repulsive region $d \lesssim \sigma$ and turns negative in the attractive region $d \geqslant \sigma$. With this definition, we can construct the friction-load curve by varying the distance $d$ for any given value of the slider speed. Figure 3 reports two such curves for $\bar{v}_{\mathrm{SL}}=0.18$ and $\bar{v}_{\mathrm{SL}}=0.7$. As shown in the inset of Fig. 3, for sufficiently large load (slider close to the substrate) a joint increase of friction $F$ and load $L$ is observed. The friction increase with load is to a good approximation represented by $F \propto L^{2}$ rather than linear with $L$ as in, e.g., Amonton's law of macroscopic friction. The reason for this quadratic friction increase with load is understood as connected to the inverse power-law repulsive behavior $\left(V_{\mathrm{ext}} \propto d^{-12}\right.$ of the LJ interaction, but the same would hold for another exponent) at short range. In this small- $d$ regime indeed $L \propto V_{\text {ext }}$, whence $F \propto\left|V_{\text {ext }}\right|^{2}$-Eq. (1)-entails $F \propto|L|^{2}$.

The additional novelty at large speed $(\gtrsim 30 \%$ of the sound velocity) is nonmonotonicity of friction versus load. In this regime, exemplified by $\bar{v}_{\mathrm{SL}}=0.7$ (squares in Fig. 3), friction initially decreases for increasing load [as also noted in Fig. 1(d)] until it nearly vanishes and then increases again. 


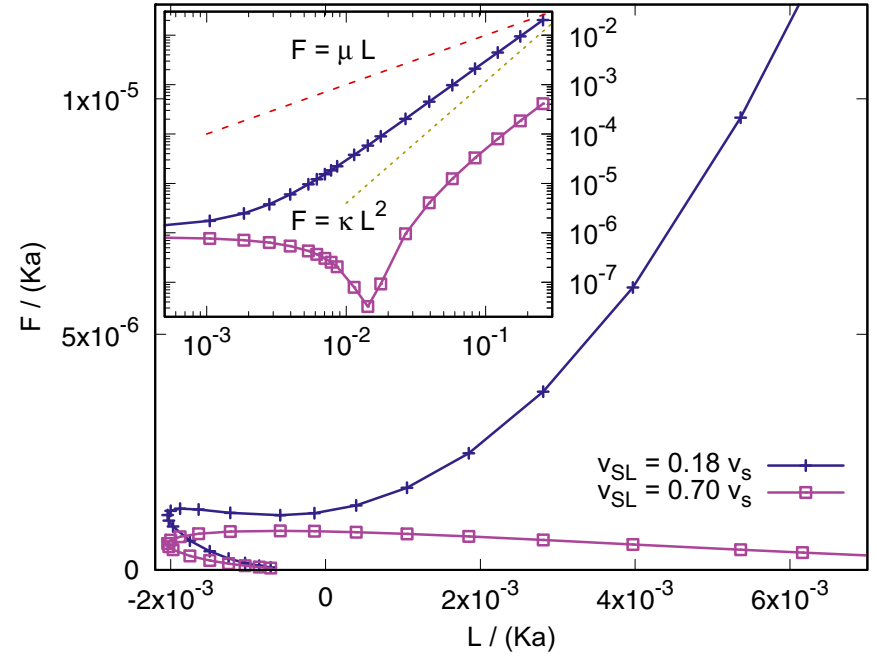

FIG. 3. Load dependence of the dynamic friction force computed according to Eq. (1) for a large range of distances at two sliding speeds. Comparison with a linear increase (Amonton-like) is also shown. The effective load $L$ is estimated as the average vertical force acting on the slider in one sliding period, Eq. (14). The model parameters are the same as for Fig. 1. The connecting lines are guides to the eye. In the inset the scale is logarithmic on both axes to better illustrate the quadratic scaling of friction for large loads, at all speeds. At small positive loads, friction becomes very nonmonotonic and even exhibits a deep minimum at the larger considered speed. The reentrance of both friction curves at negative loads exhibits the standard attractive, large-distance regime observed in AFM experiments.

The reason for this frictional dip-which would be a zero in the $\gamma \rightarrow 0$ limit-is a change of sign of the Fouriertransformed interaction $V_{\text {ext }}(Q)$ caused in turn by real-space attractive-repulsive-attractive oscillations which occur along $x$ when the load is increased in a certain range. This kind of matrix-element zero is the straight analog of the "Cooper zero" well known in atomic spectra [34]. In our case, at any velocity, the dominant contributions to friction come from the wave vectors given by Eq. (9) (and their surroundings of order $\gamma / v_{\mathrm{s}}$ ), which for $\bar{v}_{\mathrm{SL}}=0.7$ correspond to one single region around $Q \simeq 2.8204 / a$, see Figs. 1(b) and 1(c). For $d>\sigma,\left|V_{\text {ext }}(Q)\right|$ has a single peak for $Q=0$. However for $d \gtrsim \sigma$ the interaction develops a small repulsive contribution when the slider is atop a particle of the substrate, generating a second peak in $\left|V_{\text {ext }}(Q)\right|$ at some finite $Q>0$. As $d$ decreases, this second peak becomes more and more prominent, and en route there is a wavelength where $\left|V_{\text {ext }}(Q)\right|=0$. For $\bar{v}_{\mathrm{SL}}=0.7$ this antiresonance condition overlaps the resonant wave vector $Q \simeq$ $2.8204 / a$ for the value of distance $d \simeq 0.425 a$, as illustrated in Fig. 4(b). This corresponds exactly to the friction dip of Fig. 3. For $\bar{v}_{\mathrm{SL}}=0.18$, Eq. (9) yields three resonant $Q$ s, so that the vanishing of $V_{\text {ext }}(Q)$ suppresses only one of the three, with a much weaker overall effect of such antiresonance conditions.

Variations of the LJ parameter $\sigma$ characterizing the range of action of the slider-chain interaction also affect friction through changes in the Fourier transform $V_{\text {ext }}(Q)$. To investigate this effect we keep the distance at a fixed fraction $d=0.92 \sigma$, corresponding to approximately vanishing load. Whenever $\sigma$ is significantly larger than the substrate lattice parameter $a$, the

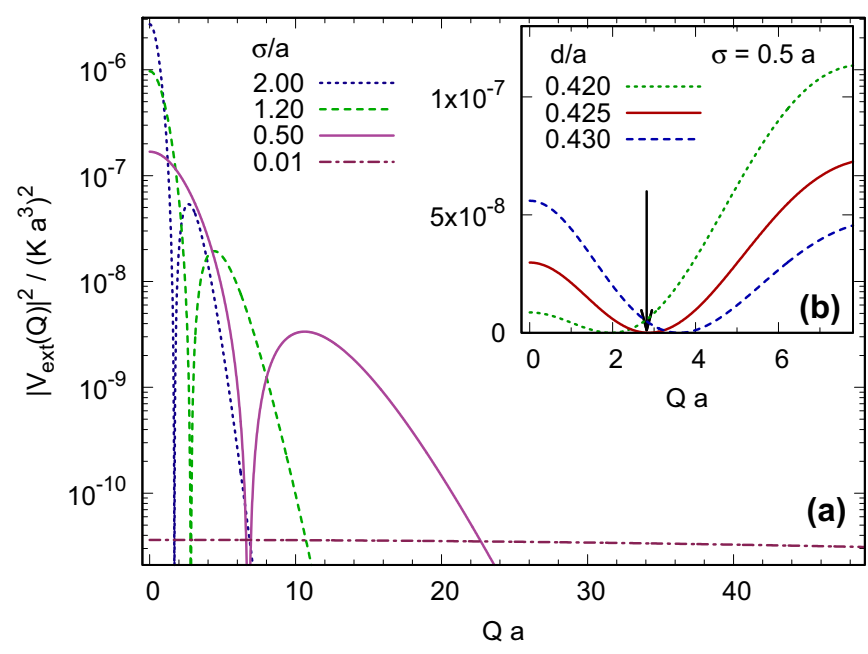

FIG. 4. The $\left|V_{\text {ext }}(Q)\right|$ "form factors" for varying LJ parameter $\sigma$ and distance $d$ in the slider-chain interaction potential, assuming an interaction depth $V_{0}=5 \times 10^{-4} \mathrm{Ka}^{2}$. (a) Distance is a fixed ratio $d=0.92 \sigma$ to the LJ parameter. (b) Detail of the region where $V_{\text {ext }}(Q)$ vanishes, for fixed $\sigma=0.5 a$, and for three similar values of $d$. The bold arrow marks the $Q$ value $2.8204 / a$ corresponding to the $v_{\mathrm{SL}}=$ $0.7 v_{\mathrm{s}}$ line crossing the dispersion in Fig. 1(b).

interaction spreads over a large number of harmonic beads, with the result that the Fourier transform of the LJ potential is non-negligible only for small wave vectors, as in the $\sigma=2.0 a$ curve of Fig. 4(a). As a result, as shown in Fig. 5, for larger $\sigma$ the only prominent resonant feature is the one near the speed of sound. By contrast if $\sigma \simeq a$ several Fourier components contribute similarly to $V_{\text {ext }}(Q)$, resulting in several visible frictional features at the resonant speeds of Table I. For $\sigma \ll a$ the slider interacts and collides with the chain particles one at a time, resulting in a sharply position-dependent potential, characterized by a slow $Q$ dependence and comparably large high- $Q$ Fourier components, as in the $\sigma=0.01 a$ (dot-dashed) curve of Fig. 4(a). As a result, dissipation picks up robust

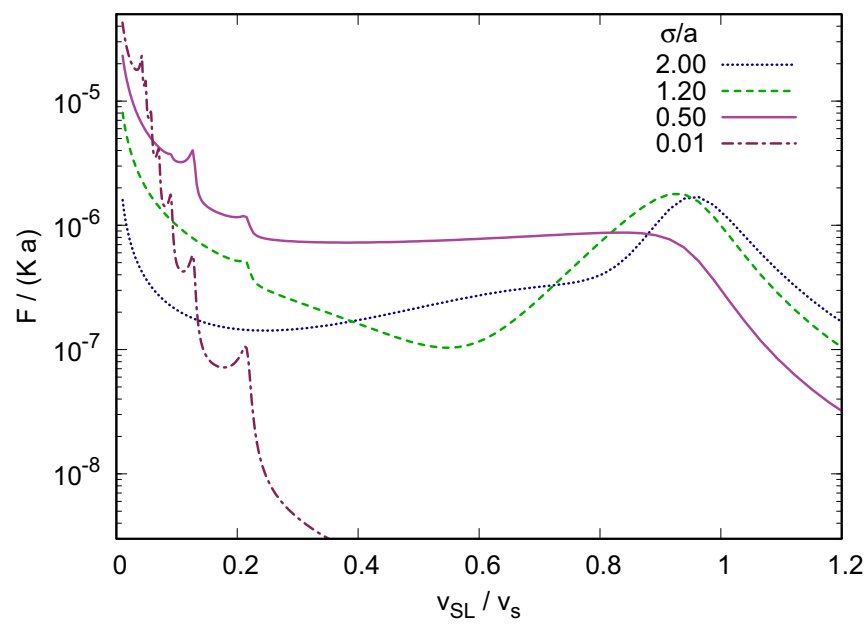

FIG. 5. Friction-velocity profiles obtained for the same $\sigma$ values as in Fig. 4. Distance is always $d=0.92 \sigma$. Other parameters are slider interaction depth $V_{0}=5 \times 10^{-4} K a^{2}$ and damping rate $\gamma=$ $0.1(m / K)^{1 / 2}$. 


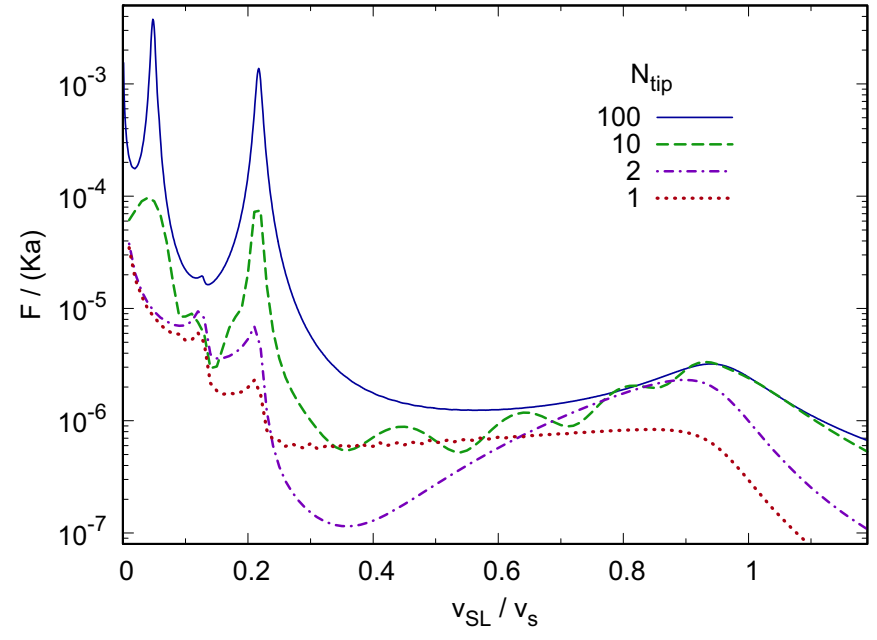

FIG. 6. Friction profile for simulated composite tips consisting in the sum of $N_{\text {tip }}$ equal LJ contributions displaced at intervals of $l=0.7 a$. All other parameters are the same as used previously: $\sigma=$ $0.5 a, d=0.465 a, V_{0}=5 \times 10^{-4} K a^{2}$, and $\gamma=0.1(m / K)^{1 / 2}$.

contributions from large wave vectors, resulting in many resonances visible at low speed (dot-dashed curve in Fig. 5).

Next, we checked the effect a different slider-chain potential form. Comparing a Morse potential characterized by the same equilibrium distance $\sigma$, potential well depth $V_{0}$, and curvature at the minimum as the $\mathrm{LJ}$ potential, the resulting frictionvelocity curves (not shown) are qualitatively and quantitatively quite similar to those of Fig. 5 for $d$ of the order of $\sigma$.

More interestingly, we can exploit the simple structure of the analytic result of Eq. (1) to investigate a basic model for a rigid extended tip. We construct one by building the external potential as the sum of $N_{\text {tip }}$ LJ potentials, placed at fixed separation $l$ one from the next:

$$
V_{\text {ext }}=\sum_{i=1}^{N_{\text {tip }}} V_{\mathrm{LJ}}\left(\left[(x-i l-v t)^{2}+d^{2}\right]^{1 / 2}\right) .
$$

For $N_{\text {tip }}=1$ we recover the pointlike slider discussed previously. In the limit $N_{\text {tip }} \rightarrow \infty$ the slider generates a periodic potential, so that the model should resemble closely the Frenkel-Kontorova model [29,30], at least for $d \gg \sigma$, where the lowest Fourier component at period $l$ would dominate the effective corrugation experienced by the chain atoms.

Here we focus on a finite-size slider, representative of a microscopic contact such as a rigid AFM tip. As an example, Fig. 6 compares the dynamic friction as a function of speed for $N_{\text {tip }}=1,2,10,100$, in the usual conditions $\sigma=0.5 a, d=$ $0.92 \sigma$, and $\gamma=0.1(m / K)^{1 / 2}$. The resulting friction curves exhibit an additional and interesting dependence upon the tip size. The already discussed $N_{\text {tip }}=1$ friction pattern is complicated by the addition of oscillations for $N_{\text {tip }}=2$ and 10 , until simplicity is again recovered when we reach $N_{\text {tip }}=100$. These features can be understood by examining the effect of increasing the number of LJ particles on the Fourier transform of the potential. As illustrated in Fig. 7, as the number of tip atoms grows, $\left|V_{\text {ext }}(Q)\right|^{2}$ exhibits an increasing number of oscillations, with $Q$-space period $2 \pi /\left[\left(N_{\text {tip }}-1\right) l\right]$, generated by the sharp edges of a slider whose overall size is $\left(N_{\text {tip }}-1\right) l$.

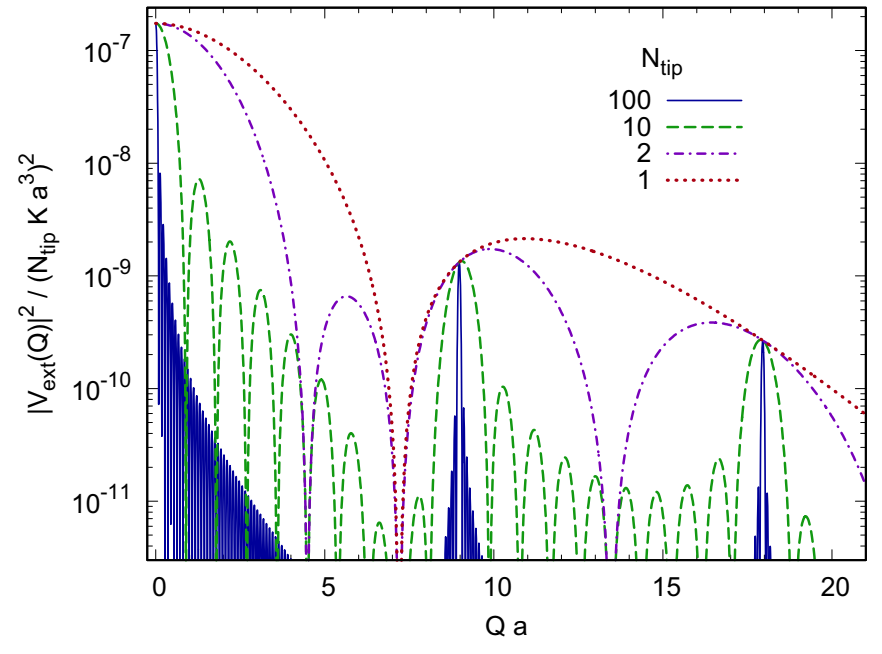

FIG. 7. Comparison of the Fourier transforms $\left|V_{\mathrm{ext}}(Q)\right|^{2}$ of the slider-chain interaction, for simulated sliders consisting in the sum of $N_{\text {tip }} \mathrm{LJ}$ contributions placed regularly at an interval $l=0.7 a$. The other parameters for $V_{\mathrm{ext}}$ are the standard ones used in this work: $\sigma=0.5 a, d=0.465 a, V_{0}=5 \times 10^{-4} K a^{2}$.

These fast oscillations produce sets of wave vectors $\left\{Q_{i}^{0}\right\}$ such that $V_{\text {ext }}\left(Q_{i}^{0}\right)=0$ : These wave vectors yield friction dips for the velocities $v_{\mathrm{SL}}$ matching the condition $Q_{i}^{0} v_{\mathrm{SL}}=\omega\left(Q_{i}^{0}\right)$. These dips are especially visible for $N_{\text {tip }}=10$, dashed line in Fig. 6. As $N_{\text {tip }}$ grows, the distance between the zeros of $V_{\text {ext }}(Q)$ decreases, the number of friction dips increases, and their visibility decreases: Beyond a certain slider size the wave vector $2 \pi /\left[\left(N_{\text {tip }}-1\right) l\right]$ of this oscillation becomes shorter than the phonon broadening, of the order of $\gamma / v_{\mathrm{s}}$, introduced by the damping term.

In addition to the size-related oscillations, for increasing $N_{\text {tip }},\left|V_{\text {ext }}(Q)\right|^{2}$ develops stronger and sharper Bragg peaks related to the periodicity of the atoms in the tip. The relevant $G$ vectors are located at integer multiples of $G_{1}=2 \pi / l$, which for the selected spacing $l=0.7 a$ is $G_{1}=\simeq 8.976 / a$, especially evident in the solid curve of Fig. 7 . The resonant strengths $\left|V_{\text {ext }}\left(G_{i}\right)\right|^{2}$ grow with $N_{\text {tip }}^{2}$-note that in Fig. $7\left|V_{\text {ext }}(Q)\right|^{2}$ is reported divided by $N_{\text {tip }}^{2}$, so that the Bragg peaks retain the same heights for all curves. These intensities are modulated by the "atomic form factor" represented by $\left|V_{\text {ext }}(Q)\right|^{2}$ for $N_{\text {tip }}=1$, namely the dotted curve of Fig. 7. For the selected spacing $l=0.7 a$, the near coincidences of $G_{1} \simeq Q_{2}$ and $G_{3} \simeq Q_{5}$, among the resonant vectors $Q_{j}$ reported in Table I, imply that the corresponding resonant peaks at $v_{\mathrm{SL} 2} \simeq 0.22 v_{\mathrm{s}}$ and $v_{\mathrm{SL} 5} \simeq 0.07 v_{\mathrm{s}}$ are especially prominent in the $F$ profile for $N_{\text {tip }}=10$ and 100 - dashed and solid curves of Fig. 6. As the spacing $l$ of the slider atoms can be controlled independently of other model parameters, the analytic formulation of the present paper provides a way to engineer a specially crafted polyatomic slider with Bragg peaks suitably placed in order to enhance or suppress specific resonances, thus tuning the speed dependence of friction practically at will.

\section{CONCLUSIONS}

Based on an exact LRT starting point and brought to a viable formula by means of well-understood approximations, 
we present an analytic result for the dynamic sliding friction in a minimal model for sliding in the weak-interaction regime. This formula provides a transparent physical decomposition of the friction force into products of equilibrium dynamical properties of the unperturbed chain and mechanical properties of the slider-chain interaction. Within this approximation, the sliding of a small rigid slider at a certain velocity excites selectively those phonons in the substrate moving at the same phase velocity. Specific resonances occur when these phonons share both the same phase velocity and group velocity as that of the slider.

Simple as this approach is, it shows two strong features. The first is that it is conceptually and practically straightforward to calculate friction [35]. The second is that at least in the test system adopted it reproduces with striking accuracy the friction observed in much more laborious numerical simulations [17], whose output serves as a validating numerical experiment.

Importantly, the analytic relation obtained provides an explicit, therefore powerful insight into weak, smooth, dry sliding friction. The explicit analytic form potentially provides an easy tuning of the properties of the sliders and their interactions with the purpose of tailoring a desired speed and load dependence of dynamical friction.

We purposely conducted this study for a simple idealized 1D model, as opposed to a more specific one, because of the clarity with which the results and phenomena could be uncovered as a function of parameters. The understanding and the stunning accuracy obtained suggest that this study may serve as a guide for future applications of this method to more realistic 3D systems. Suitable approximations to the structure factor $S_{n n}$ of 3D substrates, and of slider-substrate interaction, could be used for approximate predictions of friction for realistic interfaces, at least in the weak-interaction regime, at arbitrary speed and variable load, including regimes such as low speeds which simulations cannot reach. We should reiterate here that by its linear-response basis the method only applies to smooth sliding at finite speed, while it fails to predict anything about the transition from dynamic to static friction, and thus about intrinsically nonlinear regimes such as stick-slip. This approach on the other hand may become quite valuable in providing analytical clues to the behavior of friction including systems and circumstances where its applicability might be considered borderline.

\section{ACKNOWLEDGMENTS}

Work in Trieste was carried out under funding from the European Research Council (ERC) under the European Union's Horizon 2020 research and innovation programme (Grant Agreement No. 320796 - "MODPHYSFRICT"). The authors would also like to gratefully acknowledge networking support by the COST Action MP1303.

\section{APPENDIX A: DERIVATION OF THE LRT EXPRESSION IN EQ. (4)}

To obtain Eq. (4), we start from Eq. (3) and represent $\chi_{n n}^{R}$ as a Fourier transform. To enforce the crystalline translational invariance [36] we write:

$$
\begin{aligned}
\chi_{n n}^{R}\left(x, x^{\prime} ; t-t^{\prime}\right)= & \sum_{G} \int_{-\infty}^{+\infty} \frac{d Q}{2 \pi} \int_{-\infty}^{+\infty} \frac{d \omega}{2 \pi} \mathrm{e}^{-i \omega\left(t-t^{\prime}\right)} \\
& \times \mathrm{e}^{i Q x} \chi_{n n}^{R}(Q, Q+G ; \omega) \mathrm{e}^{-i(Q+G) x^{\prime}},
\end{aligned}
$$

where the sum over $G$ runs over the reciprocal lattice vectors $G=2 \pi n / a$. Since the only time dependence of the perturbing potential is a space-shift, $V_{\text {ext }}(x, t)=V_{\text {ext }}\left(x-v_{\mathrm{SL}} t, 0\right)$, its standard Fourier representation is:

$$
V_{\mathrm{ext}}(x, t)=\int_{-\infty}^{+\infty} \frac{d q}{2 \pi} \mathrm{e}^{i q\left(x-v_{\mathrm{SL}} t\right)} V_{\mathrm{ext}}(q) .
$$

Notice that $V_{\mathrm{ext}}(-q)=V_{\mathrm{ext}}^{*}(q)$ since $V_{\mathrm{ext}}\left(x-v_{\mathrm{SL}} t, 0\right)$ is a real function. To calculate the average dissipation we average $\dot{E}=$ $W$ in Eq. (3) over a period of time $\tau=a / v_{\mathrm{SL}}$. Inserting all Fourier transforms we have:

$$
\begin{aligned}
F\left(v_{\mathrm{SL}}\right)= & \frac{1}{v_{\mathrm{SL}}} \bar{W}=-\frac{1}{v_{\mathrm{SL}} \tau} \int_{0}^{\tau} d t \int_{-\infty}^{+\infty} d x \int_{-\infty}^{+\infty} d x^{\prime} \int_{-\infty}^{+\infty} d t^{\prime} \\
& \times \int_{-\infty}^{+\infty} \frac{d q}{2 \pi} \int_{-\infty}^{+\infty} \frac{d q^{\prime}}{2 \pi} \sum_{G} \int_{-\infty}^{+\infty} \frac{d Q}{2 \pi} \int_{-\infty}^{+\infty} \frac{d \omega}{2 \pi} \times \\
& \times \mathrm{e}^{i(q+Q) x} \mathrm{e}^{-i\left(q^{\prime}+Q+G\right) x^{\prime}} \mathrm{e}^{-i t\left(\omega+q v_{\mathrm{SL}}\right)} \mathrm{e}^{i t^{\prime}\left(\omega+q^{\prime} v_{\mathrm{SL}}\right)} \\
& \times(-i \omega) \chi_{n n}^{R}(Q, Q+G ; \omega) V_{\mathrm{ext}}(q) V_{\mathrm{ext}}^{*}\left(q^{\prime}\right) .
\end{aligned}
$$

Integration over $x$ and $x^{\prime}$ yields two Dirac-delta distributions for $q$ and $q^{\prime}$, leading to:

$$
\begin{aligned}
F\left(v_{\mathrm{SL}}\right)= & -\frac{1}{v_{\mathrm{SL}} \tau} \int_{0}^{\tau} d t \int_{-\infty}^{+\infty} d t^{\prime} \sum_{G} \int_{-\infty}^{+\infty} \frac{d Q}{2 \pi} \int_{-\infty}^{+\infty} \frac{d \omega}{2 \pi} \\
& \times \mathrm{e}^{-i t\left(\omega-Q v_{\mathrm{SL}}\right)} \mathrm{e}^{i t^{\prime}\left(\omega-(Q+G) v_{\mathrm{SL}}\right)}(-i \omega) \chi_{n n}^{R} \\
& \times(Q, Q+G ; \omega) V_{\mathrm{ext}}^{*}(Q) V_{\mathrm{ext}}(Q+G) .
\end{aligned}
$$

The integral over $t^{\prime}$ gives a Dirac delta $2 \pi \delta\left(\omega-(Q+G) v_{\mathrm{SL}}\right)$ :

$$
\begin{aligned}
F\left(v_{\mathrm{SL}}\right)= & -\frac{1}{v_{\mathrm{SL}} \tau} \sum_{G} \int_{0}^{\tau} d t \mathrm{e}^{-i t G v_{\mathrm{SL}}} \int_{-\infty}^{+\infty} \frac{d Q}{2 \pi}\left(-i(Q+G) v_{\mathrm{SL}}\right) \\
& \times \chi_{n n}^{R}\left(Q, Q+G ;(Q+G) v_{\mathrm{SL}}\right) V_{\mathrm{ext}}^{*}(Q) V_{\mathrm{ext}}(Q+G) .
\end{aligned}
$$

The $t$ integral over a period $\tau$ now involves

$$
\frac{1}{\tau} \int_{0}^{\tau} d t \mathrm{e}^{-i t G v_{\mathrm{SL}}}=\delta_{G, 0},
$$

due to $\tau=a / v_{\mathrm{SL}}$. Hence:

$$
\begin{aligned}
F\left(v_{\mathrm{SL}}\right)=- & \frac{1}{v_{\mathrm{SL}}} \int_{-\infty}^{+\infty} \frac{d Q}{2 \pi}\left(-i Q v_{\mathrm{SL}}\right) \\
& \times \chi_{n n}^{R}\left(Q, Q ; Q v_{\mathrm{SL}}\right)\left|V_{\mathrm{ext}}(Q)\right|^{2} .
\end{aligned}
$$

Equation (A4) shows that the relevant frequency $\omega$ contributing is related to $Q$ via

$$
\widetilde{\omega}_{Q}=Q v_{\mathrm{SL}} .
$$

Next, we use the standard properties of $\chi_{n n}^{R}(Q, Q ; \omega)$ to show that only the imaginary part of $\chi_{n n}^{R}$ contributes. Indeed, hermiticity of the density operator implies that $\chi_{n n}^{R}(Q, Q ; \omega)^{*}=\chi_{n n}^{R}(Q, Q ;-\omega)$ and $\chi_{n n}^{R}(Q, Q ; \omega)=$ $\chi_{n n}^{R}(-Q,-Q ; \omega)$. Since $\left|V_{\mathrm{ext}}(Q)\right|^{2}$ is an even function of $Q$, 
only the even part of $Q \chi_{n n}^{R}\left(Q, Q ; Q v_{\mathrm{SL}}\right)$ contributes to the integral. Simple algebra shows that

$$
F\left(v_{\mathrm{SL}}\right)=-\frac{2}{v_{\mathrm{SL}}} \int_{0}^{+\infty} \frac{d Q}{2 \pi} \widetilde{\omega}_{Q} \operatorname{Im} \chi_{n n}^{R}\left(Q, Q ; \widetilde{\omega}_{Q}\right)\left|V_{\mathrm{ext}}(Q)\right|^{2},
$$

which is the first form of Eq. (4).

The second form comes from the use of the fluctuationdissipation relation [19]

$$
\operatorname{Im} \chi_{n n}^{R}(Q, Q ; \omega)=-\frac{1}{2}\left(1-\mathrm{e}^{-\beta \hbar \omega}\right) S_{n n}(Q, Q ; \omega),
$$

where $S_{n n}(Q, Q ; \omega)$ is the structure factor:

$$
\begin{aligned}
S_{n n}(Q, Q ; \omega)= & \lim _{V \rightarrow \infty} \frac{1}{V} \int_{V} d x^{\prime} \int_{V} d x \mathrm{e}^{-i Q\left(x-x^{\prime}\right)} \frac{1}{\hbar} \\
& \times \int_{-\infty}^{+\infty} d t \mathrm{e}^{i \omega t}\left\langle\hat{n}(x, t) \hat{n}\left(x^{\prime}, 0\right)\right\rangle \\
= & \frac{1}{\hbar} \lim _{V \rightarrow \infty} \frac{1}{V} \int_{-\infty}^{+\infty} d t \mathrm{e}^{i \omega t}\left\langle\hat{n}_{Q}(t) \hat{n}_{-Q}(0)\right\rangle .
\end{aligned}
$$

Here

$$
\hat{n}_{Q}(t)=\int_{V} d x \mathrm{e}^{-i Q x} \hat{n}(x, t)
$$

is the Fourier transform of the density operator (in Heisenberg representation) for a system in a $1 \mathrm{D}$ "volume" $V$ with periodic boundary conditions (PBC).

\section{APPENDIX B: EVALUATION OF THE STRUCTURE FACTOR FOR THE HARMONIC CHAIN}

In this appendix we calculate the structure factor for a harmonic chain. We use the standard [28] displacement $\hat{u}_{j}(t)$ from the equilibrium position $x_{j}^{(0)}=a j$ for each atom $j$ to write the Fourier transform of the density operator as:

$$
\hat{n}_{Q}(t)=\sum_{j=1}^{N} \mathrm{e}^{-i Q a j} \mathrm{e}^{-i Q a \hat{u}_{j}(t)},
$$

where $N$ is the number of atoms, hence $V=N a$ is the volume, and $\mathrm{PBC}$ are assumed. Using this expression and the lattice translational invariance of the problem we can write the structure factor of Eq. (A8) as:

$$
\begin{aligned}
S_{n n}(Q, Q ; \omega)= & \lim _{N \rightarrow \infty} \frac{1}{\hbar a} \int_{-\infty}^{+\infty} d t \mathrm{e}^{i \omega t} \\
& \times \sum_{j=1}^{N} \mathrm{e}^{-i Q a j}\left\langle\mathrm{e}^{-i Q \hat{u}_{j}(t)} \mathrm{e}^{+i Q \hat{u}_{0}(0)}\right\rangle .
\end{aligned}
$$

To evaluate the averages, we use the known Gaussian identity $\left\langle\mathrm{e}^{\hat{A}} \mathrm{e}^{\hat{B}}\right\rangle=\mathrm{e}^{\frac{1}{2}\left\langle\hat{A}^{2}\right\rangle+\frac{1}{2}\left\langle\hat{B}^{2}\right\rangle+\langle\hat{A} \hat{B}\rangle}$ valid for harmonic-oscillator operators [37]. A direct application of this formula to our case of interest, together with translational invariance in time and lattice cell index, leads to:

$$
\left\langle\mathrm{e}^{-i Q \hat{u}_{j}(t)} \mathrm{e}^{i Q \hat{u}_{0}(0)}\right\rangle=\mathrm{e}^{-Q^{2}\left\langle\hat{u}_{j}(t) \hat{u}_{j}(t)\right\rangle} \mathrm{e}^{Q^{2}\left\langle\hat{u}_{j}(t) \hat{u}_{0}(0)\right\rangle} .
$$

By expressing the displacement operators $\hat{u}_{j}$ in terms of standard harmonic oscillators for the phonons [28]

$$
\hat{u}_{j}(t)=\frac{1}{\sqrt{N}} \sum_{k \neq 0}^{\mathrm{BZ}} \mathrm{e}^{i k a j} \sqrt{\frac{\hbar}{2 m \omega(k)}}\left(\mathrm{e}^{-i \omega(k) t} \hat{b}_{k}+\mathrm{e}^{i \omega(k) t} \hat{b}_{-k}^{\dagger}\right),
$$

we can easily calculate:

$$
\begin{aligned}
\left\langle\hat{u}_{j}(t) \hat{u}_{j}(t)\right\rangle= & \frac{1}{N} \sum_{k}^{\mathrm{BZ} \backslash\{0\}} \frac{\hbar}{2 m \omega(k)}\left[2 n_{B}(k)+1\right] \\
\left\langle\hat{u}_{j}(t) \hat{u}_{0}(0)\right\rangle= & \frac{1}{N} \sum_{k}^{\mathrm{BZ} \backslash\{0\}} \frac{\hbar}{2 m \omega(k)}\left[n_{\mathrm{B}}(k) \mathrm{e}^{i \omega(k) t}+\left(n_{\mathrm{B}}(k)+1\right)\right. \\
& \left.\times \mathrm{e}^{-i \omega(k) t}\right] \mathrm{e}^{i k a j},
\end{aligned}
$$

where $n_{B}(k)=1 /\left(\mathrm{e}^{\beta \hbar \omega(k)}-1\right)$ is the Bose distribution factor By combining Eq. (B5) with Eq. (B3), we finally arrive at the following expression for the structure factor of a harmonic chain (in the thermodynamic limit):

$$
\begin{aligned}
S_{n n}(Q, Q ; \omega)= & \frac{1}{\hbar a} \int_{-\infty}^{+\infty} d t \mathrm{e}^{i \omega t} \sum_{j=-\infty}^{+\infty} \mathrm{e}^{-i Q a j} \mathrm{e}^{-Q^{2} \Phi_{j}(t, \beta)} \\
\Phi_{j}(t, \beta)= & a \int_{-\frac{\pi}{a}}^{+\frac{\pi}{a}} \frac{d k}{2 \pi} \frac{\hbar}{2 m \omega(k)}\left\{\left[2 n_{\mathrm{B}}(k)+1\right]\right. \\
& \left.-\left[n_{\mathrm{B}}(k) \mathrm{e}^{i \omega(k) t}+\left(n_{\mathrm{B}}(k)+1\right) \mathrm{e}^{-i \omega(k) t}\right] \mathrm{e}^{i k a j}\right\} .
\end{aligned}
$$

We could find no way to evaluate this exact expression. To proceed we resort to the standard one-phonon [28] expansion of the exponential

$$
\mathrm{e}^{-Q^{2} \Phi_{j}(t, \beta)} \simeq 1-Q^{2} \Phi_{j}(t, \beta) .
$$

Such an approximation is rather drastic, especially in low dimension: Indeed, the argument $Q^{2} \Phi_{j}(t, \beta)$ of the exponential in Eq. (B7) is not always small. One can argue that the $Q$ dependence can be regularized by a sufficiently fast decay of the potential $\left|V_{\text {ext }}(Q)\right|^{2}$, thus legitimating an expansion. Nevertheless, even if $Q$ is assumed to be small one can show that in $1 \mathrm{D} \Phi_{j}(t, \beta)$ would diverge linearly in time (or logarithmically, for $T=0$ ) for large $t$. The divergence of $\Phi_{j}(t, \beta)$ leads to a factor $\exp \left(-Q^{2} \Phi_{j}(t, \beta)\right)$ which drops to zero for large $t$-hence the absence of elastic Bragg peaks, proportional to $\delta(\omega)$, in 1D-while the linearized one-phonon expression actually diverges. On one hand, such subtleties are just an artefact of the 1D toy problem we have considered and should not influence applications of our theory to more realistic situations. On the other hand, we find that even for a strictly 1D toy problem, the (inevitable) presence of dissipation provides a cure for the problem. Indeed the decaying exponential term in Eq. (10) of Sec. III, introduced to explicitly take into account dissipation, kills the divergences of $\Phi_{j}(t, \beta)$ at large $t$ for any temperature $T$. 
Consider now the resulting expression for the one-phonon inelastic structure factor:

$$
\begin{aligned}
& S_{n n}^{1-\mathrm{ph}}(Q, Q ; \omega>0) \\
& \quad=Q^{2} \int_{-\infty}^{+\infty} d t \int_{-\frac{\pi}{a}}^{+\frac{\pi}{a}} \frac{d k}{2 \pi} \frac{n_{\mathrm{B}}(k)+1}{2 m \omega(k)} \mathrm{e}^{i(\omega-\omega(k)) t} \sum_{j=-\infty}^{+\infty} \mathrm{e}^{i(k-Q) a j} .
\end{aligned}
$$

Here we have applied the approximation (B7) to Eq. (B6) and kept only the term that survives for $\omega>0$. We dropped all the terms inside the integrand which do not depend on time, as they give a vanishing contribution in $1 \mathrm{D}$, and would lead to elastic $\delta(\omega)$ contributions in dimensions $D>2$; we also dropped "counter-rotating" terms which oscillate as $\mathrm{e}^{i(\omega+\omega(k)) t}$. Now we make use of the periodic delta function, or
Dirac comb, identity:

$$
\sum_{j=-\infty}^{\infty} \mathrm{e}^{i(k-Q) a j}=\frac{2 \pi}{a} \sum_{G} \delta(k-Q-G),
$$

where $G=\frac{2 \pi n}{a}$ and $n$ is any integer. Hence, combining the integral over $k$ on the first BZ with the reciprocal-lattice $G$ summation resulting from Eq. (B9), one obtains an unrestricted integral on a variable $k^{\prime}=k-G$ spanning the extended BZ scheme, i.e., the entire $-\infty<k^{\prime}<+\infty$ range. The resulting $\delta\left(k^{\prime}-Q\right)$ identifies $k^{\prime}$ with $Q$. Eventually, performing the integral over $t$, which yields a delta function in $\omega$, we obtain:

$$
S_{n n}^{1-\mathrm{ph}}(Q, Q ; \omega>0)=\frac{\pi Q^{2}}{\operatorname{ma\omega }(Q)}\left[n_{\mathrm{B}}(Q)+1\right] \delta(\omega-\omega(Q)),
$$

which is equivalent to Eq. (5). Note that in this expression the dispersion $\omega(Q)$ is intended in the extended $\mathrm{BZ}$ scheme.
[1] F. P. Bowden and D. Tabor, The Friction and Lubrication of Solids (Oxford Univ. Press, New York, 1950).

[2] B. Persson, Sliding Friction: Physical Principles and Applications (Springer, Berlin, 2000).

[3] M. Robbins and M. Müser, Computer Simulations of Friction, Lubrication and Wear (CRC Press, Boca Raton, FL, 2001).

[4] M. H. Müser, Theory and Simulation of Friction and Lubrication (Springer, Heidelberg, 2006).

[5] C. M. Mate, Tribology on the Small Scale: A Bottom Up Approach to Friction, Lubrication, and Wear (Oxford Univ. Press, Oxford, 2008).

[6] A. Vanossi, N. Manini, M. Urbakh, S. Zapperi, and E. Tosatti, Rev. Mod. Phys. 85, 529 (2013).

[7] E. Gnecco and E. Meyer (eds.), Fundamentals of Friction and Wear on the Nanoscale 2nd ed. (Springer, Berlin, 2015).

[8] N. Manini, O. Braun, and A. Vanossi, Nanotribology: Nonlinear Mechanisms of Friction (Springer, Berlin, 2015).

[9] N. Manini, O. Braun, E. Tosatti, R. Guerra, and A. Vanossi, J. Phys.: Condens. Matter 28, 293001 (2016).

[10] L. Prandtl, Z. Angew. Math. Mech. 8, 85 (1928).

[11] G. A. Tomlinson, Philos. Mag. 7, 905 (1929).

[12] Y. Frenkel and T. Kontorova, Phys. Z. Sowjetunion 13, 1 (1938).

[13] T. Kontorova and Y. Frenkel, Zh. Eksp. Teor. Fiz. 8, 1340 (1938).

[14] L. Floría and J. Mazo, Adv. Phys. 45, 505 (1996).

[15] O. M. Braun and Y. S. Kivshar, The Frenkel-Kontorova Model: Concepts, Methods, and Applications (Springer, Berlin, 2004).

[16] C. Gardiner, Handbook of Stochastic Methods for Physics, Chemistry and the Natural Sciences (Springer, Berlin, 1985).

[17] C. Apostoli, G. Giusti, J. Ciccoianni, G. Riva, R. Capozza, R. L. Woulaché, A. Vanossi, E. Panizon, and N. Manini, Beilstein J. Nanotechnol. 8, 2186 (2017).

[18] R. Kubo, M. Toda, and N. Hashitsume, Statistical Physics II: Nonequilibrium Statistical Mechanics, Springer Series in SolidState Sciences (Springer, Berlin, 2012), Vol. 31.
[19] G. F. Giuliani and G. Vignale, Quantum Theory of the Electron Liquid (Cambridge Univ. Press, Cambridge, 2005).

[20] B. N. J. Persson, E. Tosatti, D. Fuhrmann, G. Witte, and C. Wöll, Phys. Rev. B 59, 11777 (1999).

[21] M. Kisiel, F. Pellegrini, G. Santoro, M. Samadashvili, R. Pawlak, A. Benassi, U. Gysin, R. Buzio, A. Gerbi, E. Meyer et al., Phys. Rev. Lett. 115, 046101 (2015).

[22] E. Granato and S. C. Ying, Phys. Rev. B 69, 125403 (2004).

[23] M. Urbakh, J. Klafter, D. Gourdon, and J. Israelachvili, Nature (London) 430, 525 (2004).

[24] G. E. Santoro, A. Vanossi, N. Manini, G. Divitini, and E. Tosatti, Surf. Sci. 600, 2726 (2006)

[25] A. Vanossi, N. Manini, F. Caruso, G.E. Santoro, and E. Tosatti, Phys. Rev. Lett. 99, 206101 (2007).

[26] N. Manini, G. Santoro, E. Tosatti, and A. Vanossi, J. Phys.: Condens. Matter 20, 224020 (2008).

[27] The Prandtl-Tomlinson condition $4 \pi^{2} U_{0} / K a^{2} \ll 1$ involves the ratio between the substrate corrugation energy $U_{0}$ and the typical elastic energy stored in the driving spring of stiffness $K$ when elongated over a corrugation lattice spacing $a$ [6].

[28] N. Ashcroft and M. Mermin, Solid State Physics (Holt-Saunders, Philadelphia, 1976), Appendix N.

[29] T. Strunz and F.-J. Elmer, Phys. Rev. E 58, 1601 (1998).

[30] J. A. van den Ende, A. S. de Wijn, and A. Fasolino, J. Phys. Condens. Matter 24, 445009 (2012).

[31] L. van Hove, Phys. Rev. 89, 1189 (1953).

[32] J. Lindhard, K. Dan. Vidensk. Selsk. Mat. -Fys. Medd. 28, 1 (1954).

[33] N. Cabrera, V. Celli, and R. Manson, Phys. Rev. Lett. 22, 346 (1969).

[34] U. Fano and J. Cooper, Rev. Mod. Phys. 40, 441 (1968).

[35] See Supplemental Material at http://link.aps.org/supplemental/ 10.1103/PhysRevB.97.104104 for a simple computer code to compute rapidly the quantities introduced in the present paper.

[36] G. Strinati, Riv. Nuovo Cimento 11, 1 (1988).

[37] N. D. Mermin, J. Math. Phys. 7, 1038 (1966). 